\title{
Effects of performing hip abduction and adduction during bridging exercise on trunk and lower extremity muscle activity in healthy individuals
}

\author{
Joo Young Hwang ${ }^{a}$, Woo Young Ahn ${ }^{a}$, Hyo Jae Kim ${ }^{a}$, Je Hyun Woo ${ }^{a}$, Woo Jin Choi ${ }^{a}$, Jae Wook Park \\ Mi Young Lee $e^{a, b}$
}

${ }^{a}$ Department of Physical Therapy, College of Health and Welfare, Sahmyook University, Seoul, Republic of Korea

'Institute of Women's Health Physical Therapy, Sahmyook University, Seoul, Republic of Korea

Objective: To investigate the effect of performing three different bridge exercise conditions on the activities of four different muscles using surface electromyography (sEMG) in healthy young adults.

Design: Cross-sectional study.

Methods: A total of 20 healthy young adults (10 males, 10 females) voluntarily participated in this study. All subjects randomly performed three different bridge conditions as follows: general bridge exercise, isometric hip abduction (IHAB) with a blue Theraband (Hygenic Corp., USA), and isometric hip adduction (IHAD) with a Swiss ball (Hygenic Corp.). The muscle activities of bilateral erector spinae (ES), gluteus maximus (GM), biceps femoris (BF), and external oblique (EO) muscles during the bridge exercises were measured using sEMG. Subjects performed each of the three bridge conditions three times in random order and mean values were obtained.

Results: For bilateral ES and BF, there was a significant increase in muscle activity in the IHAD condition compared to the general bridge and IHAB condition $(p<0.05)$. For bilateral GM, there was a significant increase in muscle activity in the IHAB condition compared to the general bridge condition $(p<0.05)$ and there was a significant increase in muscle activity in the IHAB condition compared to IHAD condition $(p<0.05)$. For left EO, a significant increase was observed in the IHAD condition compared to the general bridge condition $(p<0.05)$.

Conclusions: $\mathrm{ES}$ and BF muscle activity increases were observed with hip adduction and increased GM activity was observed with hip abduction. These findings may be applicable within the clinical field for selective trunk and lower extremity muscle activation and advanced rehabilitation purposes.

Key Words: Abdominal oblique muscles, Electromyography, Exercise, Hip, Lower extremity

\section{Introduction}

Lumbar stabilization exercises are aimed at control of forces that may cause postural instability and at maintenance of normal spinal posture for maximum conscious or unconscious adaptation in response to external loads [1]. As a closed kinetic chain exercise, bridge exercises are used for the stabilization of the lumbar and the trunk in addition to improving the muscular strength of the pelvic and lower limb muscles, and improving trunk flexor and extensor muscle control [2]. In addition, bridge exercises are used in physical therapy rehabilitation to promote trunk stabilization, relieve pressure on the buttocks, promote bed mobility, improve sit-to-stand performance, and assist in pelvic movement, which is all considered as important for walking and general functional movement [3].

Received: 27 February, 2017 Revised: 13 March, 2017 Accepted: 17 March, 2017

Corresponding author: Mi Young Lee

Department of Physical Therapy, College of Health and Welfare, Sahmyook University, 815 Hwarang-ro, Nowon-gu, Seoul 01795, Republic of Korea Tel: 82-2-3399-1629 Fax: 82-2-3399-1639 E-mail: mylee@syu.ac.kr

(c) This is an Open-Access article distributed under the terms of the Creative Commons Attribution Non-Commercial License (http://creativecommons.org/licens es/by-nc/4.0) which permits unrestricted non-commercial use, distribution, and reproduction in any medium, provided the original work is properly cited.

Copyright @ 2017 Korean Academy of Physical Therapy Rehabilitation Science 
Recent studies have applied hip movements to bridge exercises to investigate its effects on muscle activity. Kang et al. [4] compared the effects of performing bridge exercises with 0,15 , and 30 degrees of hip abduction on the gluteus maximus (GM) and hamstring (HAM) muscles in healthy subjects. The greatest amount of GM activity was observed with 30 degrees of hip abduction, and 0 degrees of hip abduction for the HAM.

A study by Choi et al. [5] had twenty-seven healthy subjects perform five different bridge conditions to investigate its effects on the erector spinae (ES), external oblique (EO), $\mathrm{GM}$, and biceps femoris (BF). Their findings included significant increases in EO muscle activation during a one-legged bridge with hip abduction and sling compared with the one-legged abduction bridge exercise, significant increases in ipsilateral GM and BF during the one-legged bridge with hip abduction compared with the one-legged bridge exercise, and significant increases in contralateral GM and BF with the one-legged bridge with hip abduction compared with the general bridge exercise. Choi et al. [6] conducted a study on the inclusion of isometric hip abduction (IHAB) with use of a Thera-band (Hygenic Corp., Akron, OH, USA) during bridge exercises to observe GM and HAM muscle activity in healthy subjects. They had found significantly greater GM muscle activity with the application of IHAB compared to without IHAB, but no significant differences in HAM muscle activity. In contrast, Jang et al. [7] investigated the effects of performing bridge exercises with hip adduction on the abdominal and hip extensor muscles in healthy female subjects. Results showed that the application of hip adduction increased muscle activities of the rectus abdominus (RA), EO, internal oblique (IO), and GM muscles during bridge exercises. Several studies have investigated the effects of including either hip abduction or hip adduction with bridge exercises on muscle activity. However, research that directly compares muscle activity with hip abduction and adduction on trunk, gluteal, and lower extremity muscles are insufficient. Therefore, the purpose of this study was to investigate the effects of performing general bridge, bridge with $\mathrm{IHAB}$, and bridge with isometric hip adduction (IHAD) exercises on the muscle activities of the ES, GM, $\mathrm{BF}$, and $\mathrm{EO}$ using surface electromyography ( $\mathrm{EMG}$ ) in healthy young adults.

\section{Methods}

\section{Subjects}

This was a cross-sectional study with 20 healthy subjects (10 males, 10 females) who were recruited from Sahmyook University with an average age of $22.85 \pm 2.85$ years, height of $169.1 \pm 9.1 \mathrm{~cm}$, and weight of $59.95 \pm 14.95 \mathrm{~kg}$. Subjects were informed of the study procedures and provided an informed consent prior to participating in this study. The exclusion criteria were as follows: (1) range of motion limitations of bilateral hip, knee, and ankle joints; (2) history of low back pain or lower extremity dysfunction, such as iliotibial band friction syndrome, patellofemoral pain syndrome, anterior cruciate ligament sprains, or chronic ankle instability within the previous 12 months [8-12]; (3) iliopsoas, rectus femoris, or tensor fasciae latae tightness as observed by the Thomas test, Ely's test, or modified Ober's test, respectively [13,14]; and (4) lumbopelvic instability shown by performing the active straight leg raise test with a pressure biofeedback unit $[15,16]$.

\section{Surface electromyographic and data processing}

Electromyographic data were collected using a wireless TeleMyo DTS (Noraxon Inc., Scottsdale, AZ, USA) and Myo-Research Master Edition 1.06 XP software (Noraxon Inc.) was used for analyzing EMG data. The EMG signals were sampled at a frequency of $1,000 \mathrm{~Hz}$, a band pass filter of between 20 and $450 \mathrm{~Hz}$, and a preset notch filter to reject $60 \mathrm{~Hz}$. The raw data were processed into the root mean square with a window of $50 \mathrm{~ms}$. Shaving of the electrode sites were performed with rubbing alcohol used subsequently to reduce impedance o the skin. Two surface electrodes were placed within a distance of $2 \mathrm{~cm}$ and were placed on the upper fibers of GM, ES, EO, and BF muscles bilaterally. Electrodes were positioned in the center of the muscle belly and parallel to the muscle fibers $[4,17,18]$. The electrode for the upper GM was placed half the distance between the trochanter and sacral vertebrae in the middle of the muscle at an oblique angle at the level of the trochanter [17]. For the ES, the electrodes were attached parallel to the spine at the level of the iliac crest, approximately $2 \mathrm{~cm}$ apart from the spine over the muscle mass. For the EO, electrodes were attached slightly above the umbilicus, midway between the anterior superior iliac spine (ASIS) and the rib cage [19]. For the BF, especially long head, electrodes were placed at midpoint between the fibular head and ischial tuberosity. Mean and maximum values of the EMG data were obtained. The 
maximal voluntary isometric contraction (MVIC) was recorded and obtained using manual muscle test (MMT) positions that were consistent with those described by Kendall et al. [13]. To collect MVIC data, subjects maintained the MMT position of each tested muscle against manual resistance for 5 seconds, three times, which was measured using a metronome (SDM-300; Samick Corp., Eumseong, Korea). All EMG data were recorded for 5 seconds, three times. With the exclusion of the first and fifth second, the middle 3 -second time interval of the contraction was used for data analysis, and a 30 -second rest period was provided between the three trials [20,21]. The mean values from the middle 3 seconds during the maximal contraction of each muscle was obtained as the MVIC EMG amplitudes of the ES, GM, BF, and EO muscles were represented as \%MVIC values for normalization.

\section{Exercise procedures}

Subjects performed the five different bridge conditions through random selection. On the mat surface, a starting position of 30 degrees of hip abduction [4] was marked with tape by the experimenter. To ensure the performance of 30 degrees of hip abduction, a goniometer was used by placing the stationary arm on the researcher's bilateral ASIS and the moving arm at the center of the patella to create 30 degrees of hip abduction. By using a goniometer, to set up 30 degrees in total for the hip abduction 30 degrees posture, the stationary arm was placed on both sides of the ASIS and then observers placed the moving arm to create a reference point, which was the center of patella to make 15 degrees. Subjects were asked to maintain 90 degrees of knee flexion white maintaining 30 degrees of hip abduction. During the general bridge position, hip extension was maintained at zero degrees. Subjects were asked to maintain each bridge con- dition for 5 seconds at the sound of the metronome and verbal instruction. To reduce muscle fatigue, subjects were given a 30-second rest period between the three trials for each condition and a 2-minute rest period between the five conditions.

\section{Bridge exercise with general position}

The subjects were positioned in supine with their feet set apart as wide as their hip joints parallel to the midline, and knee joints flexed at 90 degrees. Subjects were asked to rest their arms rest on their chest to minimize unnecessary use of arms during the bridge conditions. Two vertical metal poles (canes) were placed on the lateral sides of each knee in order to prevent hip abduction beyond 30 degrees. The canes were located at the level between the lateral femoral condyle and greater trochanter when subjects performed 30 degrees of hip abduction with 0 degrees of hip extension. Trunk and pelvis were also aligned at this point. With the lateral knees contacting the canes, subjects were asked to lift up the buttocks. When bilateral thighs reached each metal bar, subjects were asked to pause and maintain the bridge position for 5 seconds (Figure 1).

\section{Bridge exercise with isometric hip abduction}

Under the same conditions as the general bridge condition, an addition of a blue Thera-band was wrapped around the subject's bilateral distal femur to provide constant resistance. In the hook-lying position, tension of the Theraband was set to enable the subject to accomplish and maintain 30 degrees of hip abduction. Subjects maintained the bridge exercise with isometric hip abduction for 5 seconds after both metal bars were reached by the thighs (Figure 2).

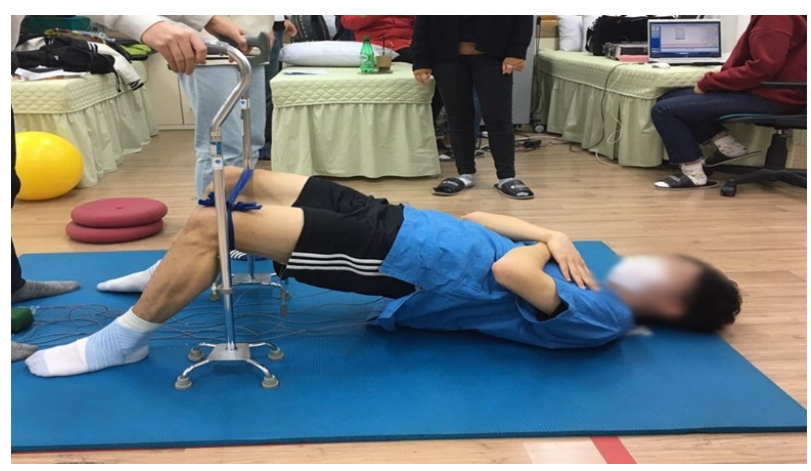

Figure 2. Bridge exercise with isometric hip abduction.
Figure 1. General bridge condition.

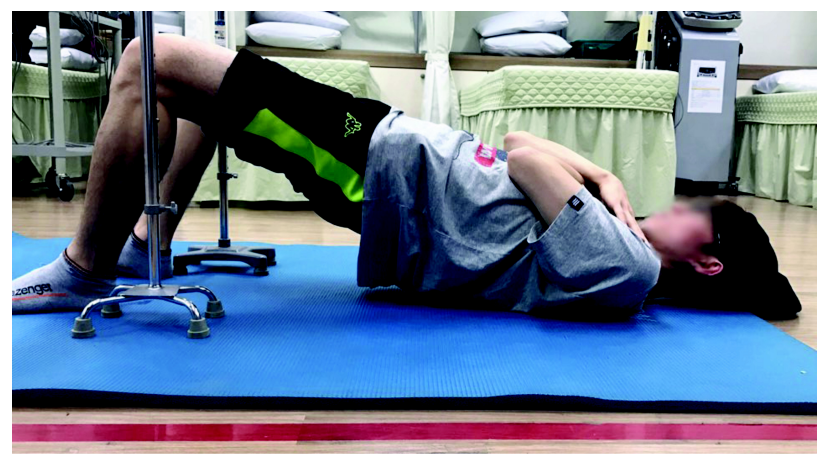




\section{Bridge exercise with isometric hip adduction}

Under the same starting condition as the general bridge condition, a Swiss ball (Hygenic Corp.) was placed between the subject's knees while maintaining 30 degrees of hip adduction. Subjects were asked to perform 30 degrees of hip adduction with the Swiss ball. To ensure 30 degrees of hip adduction was accomplished, the measurement was marked with tape on the mat. However, if 30 degrees of hip adduction could not be accomplished, the position of the ball was adjusted.

Upon lifting the buttocks and without dropping the ball, subjects were ask maintain the position for 5 seconds (Figure 3).

\section{Statistical analysis}

All statistical analysis were performed using PASW Statistics ver. 18.0 (IBM Co., Armonk, NY, USA). A one-way repeated-measures analysis of variance was used to access the statistical significance of the ES, GM, BF, and EO muscle activity during each of the five bridge conditions. Testretest reliability of EMG measurements in the three bridge exercise conditions was assessed by the intra-class correlation, $95 \%$ confidence interval, the standard error of measurement, and minimal detectable change. Statistical significance was set at 0.05 .

\section{Results}

For bilateral ES, there was a significant increase in muscle activity in the IHAD condition compared to the general bridge condition and a significant increase in muscle activity in IHAD compared to the IHAB condition $(p<0.05)$. For bilateral GM, there was a significant increase in muscle activ-

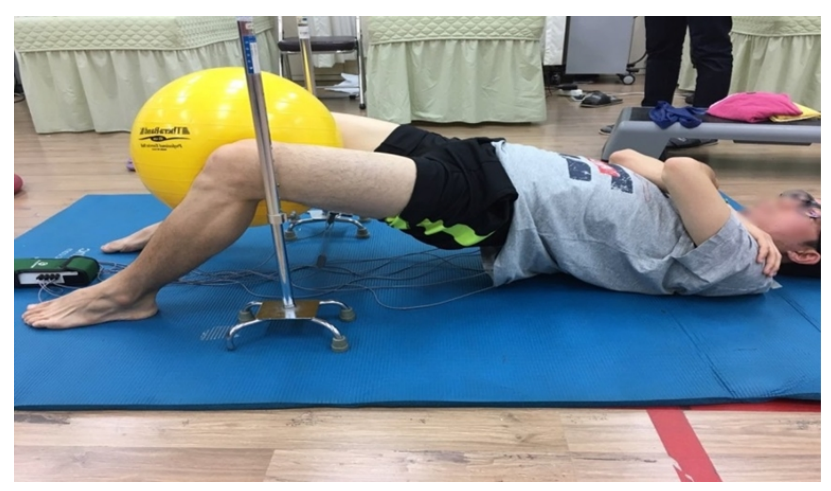

Figure 3. Bridge exercise with isometric hip adduction.

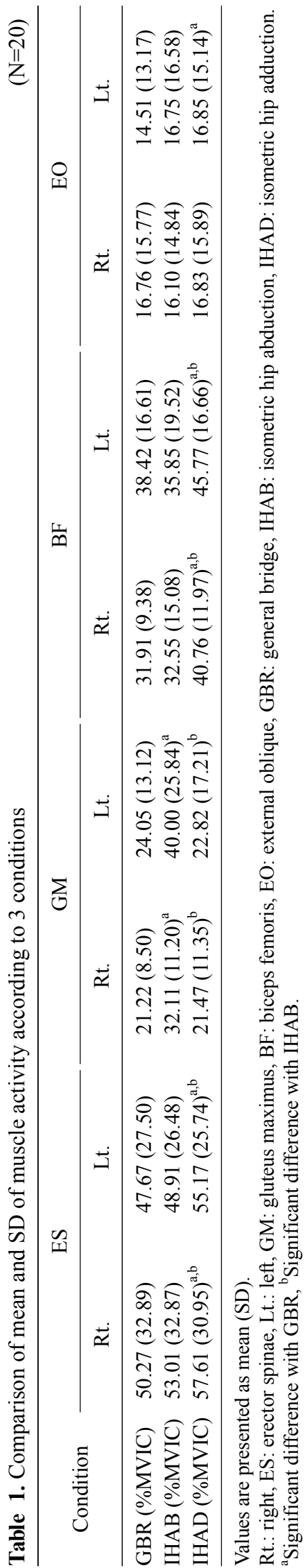


ity in the IHAB condition compared to the general bridge condition $(p<0.05)$. In contrast, there was a significant decrease in muscle activity in the IHAD condition compared to IHAB condition $(p<0.05)$. For bilateral BF, there was a significant increase in muscle activity in the IHAD condition compared to the general bridge condition $(p<0.05)$. Significant increases were also observed in the IHAD compared to the IHAB condition $(p<0.05)$. For left EO, a significant increase was observed in the IHAD condition compared to the general bridge condition $(p<0.05)$ (Table 1$)$.

\section{Discussion}

The purpose of this study was to investigate the effect of performing three different bridge exercise conditions, which included the general bridge, IHAB bridge, IHAD bridge onditions on the muscle activities of four different muscles using sEMG. Increased ES muscle activity was observed with the application of isometric hip adduction. This finding was similar with a previous study by Lee et al. [22] where ES activity was significantly higher during hip joint adduction than abduction during bridge exercises despite the sling height differences. Na et al. [23] stated that trunk muscle activity is affected by simultaneous contraction of the hip joint adductors and thus increases local muscle activity in adults in their twenties and thirties. Kang et al. [4] compared ES and GM muscle activity with increasing angles of hip abduction with bridge exercises and reported decreased ES muscle activity with increasing hip abduction angles. In contrast, greater amount of GM activity was observed with greater hip abduction angles, with the highest amount of activity found in 30 degrees of hip abduction. The results of the present study has also shown an increase in GM activity with IHAB compared to IHAD and the general bridge condition. Considering that the GM is a fusiform muscle, optimal muscle activation can be achieved when the muscle fibers are in line with the line of pull of the muscle [24]. Therefore, hip abduction during bridge exercises may put the GM in the direction of the line of pull of the muscle, which may explain the heightened muscle activity of the GM. Kang et al. [4] has also stated that performing hip abduction during hip extension may selectively increase GM activity, which was observed in this study with the isometric hip abduction bridge condition.

Kang et al. [4] reported increasing GM muscle activity associated with greater hip abduction angles, with the highest amount of activity found in 30 degrees of hip abduction but with prone hip extension with knee flexion in an open kinetic chain condition. A significant increase in left EO was observed with the IHAD condition only, although no significant differences were found in the right EO. Jang et al. [7] investigated the effects of performing bridge exercises with hip adduction on the EO, RA, IO, and GM and reported significant increases in all muscle activity with hip adduction applied compared to the general bridge exercises in healthy female subjects. It has been stated that the hip adductors originate proximal to the inferior aspect of the body and ischium and insert distally on the femur, which may affect the control of trunk muscles attached to the pelvis. They are linked to the trunk muscles to support the trunk or fix the trunk muscles and play a role in promoting contraction of the internal abdominal muscles $[25,26]$. Hip adductor contraction appears to contribute to the increased abdominal muscle activity during bridging. Although the abdominal muscle, EO, was not directly studied, Lee et al. [22] reported in their study that muscle activities of the trunk and abdominal muscle activities were higher during bridge exercises with use of a sling and during hip adduction because the contraction of the adductor magnus reduces the intra-pelvic space, which adjusts the joint locations and provides an advantageous environment for abdominal muscle contraction.

Limitations were that this study involved twenty healthy young subjects. Therefore, due to the relative small sample size and involvement of healthy subjects, it would make it difficult to apply the results to the healthy, elderly population as well as the patient population. Therefore, further research is needed to confirm the effects of isometric hip abduction and adduction on lumbar, abdominal, gluteal, and lower extremity muscle activation, especially for patients with complaints of low back and core muscle abnormalities.

This study examined the effects of performing a general bridge, bridge with IHAB, and bridge with IHAD conditions on bilateral ES, GM, BF, and EO muscle activities through the use of sEMG. Bilateral ES and BF, and left EO muscle activity increases were observed with the IHAD condition and increased bilateral GM activity was observed with the IHAB condition. These findings may be applicable within the clinical field for selective trunk and lower extremity muscle activation and advanced rehabilitation purposes.

\section{Conflict of Interest}

The authors declared no potential conflicts of interest with respect to the authorship and/or publication of this 
article.

\section{References}

1. Lee S, Park J, Lee D. Effects of bridge exercise performed on an unstable surface on lumbar stabilizing muscles according to the knee angle. J Phys Ther Sci 2015;27:2633-5.

2. Kisner C, Colby LA. Therapeutic exercise: foundations and techniques. 4th ed. Philadelphia, PA: F.A. Davis; 2002. p. 497$500,657-9$.

3. O'Sullivan SB, Schmitz TJ, Fulk G. Physical rehabilitation. 6th ed. Philadelphia, PA: F.A. Davis; 2013.

4. Kang SY, Choung SD, Jeon HS. Modifying the hip abduction angle during bridging exercise can facilitate gluteus maximus activity. Man Ther 2016;22:211-5.

5. Choi K, Bak J, Cho M, Chung Y. The effects of performing a one-legged bridge with hip abduction and use of a sling on trunk and lower extremity muscle activation in healthy adults. J Phys Ther Sci 2016;28:2625-8.

6. Choi SA, Cynn HS, Yi CH, Kwon OY, Yoon TL, Choi WJ, et al. Isometric hip abduction using a Thera-Band alters gluteus maximus muscle activity and the anterior pelvic tilt angle during bridging exercise. J Electromyogr Kinesiol 2015;25:310-5.

7. Jang EM, Kim MH, Oh JS. Effects of a bridging exercise with hip adduction on the EMG activities of the abdominal and hip extensor muscles in females. J Phys Ther Sci 2013;25:1147-9.

8. Cichanowski HR, Schmitt JS, Johnson RJ, Niemuth PE. Hip strength in collegiate female athletes with patellofemoral pain. Med Sci Sports Exerc 2007;39:1227-32.

9. Fredericson M, Cookingham CL, Chaudhari AM, Dowdell BC, Oestreicher N, Sahrmann SA. Hip abductor weakness in distance runners with iliotibial band syndrome. Clin J Sport Med 2000; 10:169-75.

10. Friel K, McLean N, Myers C, Caceres M. Ipsilateral hip abductor weakness after inversion ankle sprain. J Athl Train 2006;41: 74-8.

11. Hewett TE, Myer GD, Ford KR. Anterior cruciate ligament injuries in female athletes: Part 1, mechanisms and risk factors. Am J Sports Med 2006;34:299-311.

12. Ireland ML, Willson JD, Ballantyne BT, Davis IM. Hip strength in females with and without patellofemoral pain. J Orthop Sports
Phys Ther 2003;33:671-6.

13. Kendall FP, McCreary EK, Provance PG, Rodgers MM, Romani WA. Muscles: testing and function with posture and pain. 5th ed. Baltimore, MD: Lippincott Williams \& Wilkins; 2005.

14. Magee DJ. Orthopedic physical assessment. 5th ed. St. Louis, MO: Saunders; 2007.

15. Liebenson C. The relationship of the sacroiliac joint, stabilization musculature, and lumbo-pelvic instability. J Bodyw Mov Ther 2004;8:43-5.

16. Mens JM, Vleeming A, Snijders CJ, Stam HJ, Ginai AZ. The active straight leg raising test and mobility of the pelvic joints. Eur Spine J 1999;8:468-73.

17. Criswell E, Cram JR. Cram's introduction to surface electromyography. 2nd ed. Sudbury, MA: Jones and Bartlett Publishers; 2011.

18. Netter FH. The ciba collection of medical illustrations: vol 8: musculoskeletal system: part I: anatomy, physiology and metabolic disorders. Lincoln: Anybook Ltd; 1987.

19. Cram JR, Kasman GS, Holtz J. Introduction to surface electromyography. Gaithersburg, MD: Aspen Publishers Inc; 1998.

20. Bolgla LA, Uhl TL. Reliability of electromyographic normalization methods for evaluating the hip musculature. J Electromyogr Kinesiol 2007;17:102-11.

21. Bolgla LA, Malone TR, Umberger BR, Uhl TL. Reliability of electromyographic methods used for assessing hip and knee neuromuscular activity in females diagnosed with patellofemoral pain syndrome. J Electromyogr Kinesiol 2010;20:142-7.

22. Lee D, Park J, Lee S. Effects of bridge exercise on trunk core muscle activity with respect to sling height and hip joint abduction and adduction. J Phys Ther Sci 2015;27:1997-9.

23. Na SW, Oh DW, Park HJ. Effect of hip adductor co-contraction on trunk muscle activation during bridge exercise in healthy young individuals. J Korean Soc Phys Med 2012;7:275-82.

24. Soderberg GL. Muscle mechanics and pathomechanics. Their clinical relevance. Phys Ther 1983;63:216-20.

25. Bø K, Stien R. Needle EMG registration of striated urethral wall and pelvic floor muscle activity patterns during cough, Valsalva, abdominal, hip adductor, and gluteal muscle contractions in nulliparous healthy females. Neurourol Urodyn 1994;13:35-41.

26. Kibler WB, Chandler TJ, Livingston BP, Roetert EP. Shoulder range of motion in elite tennis players. Effect of age and years of tournament play. Am J Sports Med 1996;24:279-85. 